\title{
Noggin acts downstream of Wnt and Sonic Hedgehog to antagonize BMP4 in
}

\section{avian somite patterning}

\author{
Estelle Hirsinger ${ }^{1}$, Delphine Duprez ${ }^{2}$, Caroline Jouve ${ }^{1}$, Pascale Malapert ${ }^{1}$, Jonathan Cooke $^{3}$ and \\ Olivier Pourquié $1, *$
}

${ }^{1}$ Institut de Biologie du Développement de Marseille, LGPD-UMR CNRS 9943 Campus de Luminy - case 907, 13288 Marseille cedex 9 , France

2Institut d'Embryologie Cellulaire et Moléculaire du Centre National de la Recherche Scientifique et du Collège de France, 49 bis avenue de la Belle Gabrielle, 94736 Nogent sur Marne Cedex, France

${ }^{3}$ Division of Developmental Neurobiology, National Institute for Medical Research, The Ridgeway, Mill Hill, London NW7 1AA, UK

*Author for correspondence (e-mail: pourquie@ibdm.univ-mrs.fr)

\section{SUMMARY}

In the vertebrate embryo, the lateral compartment of the somite gives rise to muscles of the limb and body wall and is patterned in response to lateral-plate-derived BMP4. Activation of the myogenic program distinctive to the medial somite, i.e. relatively immediate development of the epaxial muscle lineage, requires neutralization of this lateral signal. We have analyzed the properties of molecules likely to play a role in opposing lateral somite specification by BMP4. We propose that the BMP4 antagonist Noggin plays an important role in promoting medial somite patterning in vivo. We demonstrate that Noggin expression in the somite is under the control of a neural-tube-derived factor, whose effect can be mimicked experimentally by Wnt1. Wnt1 is appropriately expressed in the neural tube. Furthermore, we show that Sonic Hedgehog is able to activate ectopic expression of Noggin resulting in the blocking of BMP4 specification of the lateral somite. Our results are consistent with a model in which Noggin activation lies downstream of the SHH and Wnt signaling pathways.

Key words: paraxial mesoderm, neural tube, lateral plate, muscle lineages, somite patterning, chick, Noggin, Wnt, Sonic Hedgehog

\section{INTRODUCTION}

Somites are transient mesodermal structures which gives rise to all body skeletal muscles, the dermis of the back and the axial skeleton of vertebrates (Christ and Ordahl, 1995). In amniotes, the somites appear as epithelial balls that bud off from the unsegmented or presomitic paraxial mesoderm on both sides of the neural tube. As differentiation proceeds, the ventral part of this epithelial ball becomes mesenchymal and forms the sclerotome which yields the axial skeleton whereas the still epithelial, dorsal part of the somite, the dermomyotome, then gives rise to dermis and muscle. The dermomyotome can be subdivided into a medial and a lateral compartment from which the two sets of striated muscles of the body arise: the epaxial muscles, corresponding to the deep muscles of the back, and the hypaxial muscles, which include the limb, girdle and body wall muscles (Selleck and Stern, 1991; Ordahl and Le Douarin, 1992). Switch-graft experiments have shown that, when the somite forms, its cells are not committed to particular fates in either the mediolateral or the dorsoventral dimensions (Ordahl and Le Douarin, 1992; Aoyama and Asamoto, 1988; Christ et al., 1992). This suggests that the newly formed somites are not yet determined along the mediolateral axis, and that the determination of medial and lateral somitic compartments occurs after somite formation in response to extrinsic cues provided by the surrounding envi- ronment. Structures such as the lateral plate, the overlying ectoderm and the axial organs, which include the neural tube and the notochord, have been shown to play an important role in specifying the medial and lateral compartments (Pourquié et al., 1995, 1996; Cossu et al., 1996; Pownall et al., 1996).

Some of the molecules involved in somite compartment specification have recently been identified. The TGF $\beta$ family member BMP4, known to be involved in several inductive events during embryonic development (see Hogan, 1996, for a review) is produced by the lateral plate and has been proposed to play an important role in lateral somite specification (Pourquié et al., 1996). Implantation of BMP4-expressing cells next to the prospective medial somite was shown to convert these cells to a lateral fate as defined by loss of expression of the medial marker MyoD and the ectopic expression of lateral markers such as Sim1. This lateralizing effect is antagonized by secreted factor(s) produced by the neural tube, which restrict the medial extension of the Sim1-positive domain (Pourquié et al., 1996). These axial factors, however, have not been characterized at the molecular level.

In Xenopus, BMP4 is thought to be involved in ventral mesoderm specification (see Graff, 1997, and references therein). Specification of the dorsal mesodermal lineages, including muscle and notochord, requires the inhibition of BMP4 activity by proteins such as Noggin, Chordin or Follistatin. These molecules have been shown to directly antagonize 
BMP4 by binding to this protein and preventing an interaction with its receptor (Piccolo et al., 1996; Zimmerman et al., 1996). The mediolateral determination of the avian somite presents some similarities with mesoderm patterning in frog: (i) it occurs in response to BMP4 signaling and (ii) the effect of BMP4 is antagonized by factor(s) produced by a dorsal structure, the neural tube. Therefore, the avian homologs of the molecules produced by the Spemann organizer, such as Noggin or Chordin, are potential candidates for mediating the inhibition of the BMP4 pathway in response to neural tube signals. Noggin is of particular interest since it is expressed in the dorsal neural tube in Xenopus (Smith and Harland, 1992), whereas Chordin expression has only been reported in the developing notochord (Sasai et al., 1994).

Medial somite patterning results in the early onset of the myogenic program dorsally and sclerotome formation ventrally. These developmental processes are thought to be controlled by multiple factors that include Sonic Hedgehog (SHH) and Wnt proteins, mostly produced by the axial tissues (Rong et al., 1992; Pourquié et al., 1993; Brand-Saberi et al., 1993; Goulding et al., 1994; Münsterberg and Lassar, 1995; Buffinger and Stockdale, 1994; Stern et al., 1995; Cossu et al., 1996). Several members of the Wnt family of secreted proteins are produced by the neural tube (Hollyday et al., 1995). Wnt1, Wnt3A and Wnt4 have recently been shown to play an important role in promoting myogenesis in the somite, when acting in concert with SHH (Münsterberg et al., 1995; Stern et al., 1995; Maroto et al., 1997). However, to date, no evidence exists that these factors act to antagonize BMP4.

$\mathrm{SHH}$ is produced by the developing notochord and floor plate (Riddle et al., 1993; Echelard et al., 1993; Krauss et al., 1993). It is involved in both patterning the ventral aspect of the medial somite, where it regulates the expression of the sclerotomal marker Pax-1 (Fan and Tessier-Lavigne, 1994; Fan et al., 1995; Chiang et al., 1996), and in the initial activation of the myogenic program (Münsterberg et al., 1995). Therefore, since SHH is able to mediate its effect on the unsegmented paraxial mesoderm over a considerable distance (Fan and Tessier-Lavigne, 1994), floor-plate-derived SHH is a potential candidate for mediating the effect of the neural tube on mediolateral somite specification. In addition, $\mathrm{SHH}$ was shown to be important for dorsoventral patterning of the neural tube by opposing the action of BMP4 produced dorsally (Liem et al., 1995), though it is unclear whether this is by direct action on target gene expression or by activation of a gene whose product antagonizes BMP-receptor interaction.

In this report, we have examined the role of the potential axial BMP4 antagonists, Noggin, SHH and Wnts, in the mediolateral patterning of somites. We found Noggin expression during avian somitogenesis to be compatible with an antagonizing role to BMP4. Soon after somite formation, Noggin and BMP4 are expressed in the medial dermomyotome and the lateral plate, respectively. We show that Noggin is able to antagonize the lateralizing effect of lateralplate-derived BMP4. We further demonstrate that a neuraltube-derived signal is required for Noggin expression in the medial somite. We show that Wnt1 can mimick this signal and rescue Noggin expression in the medial somite. These experiments favour a relay system of signaling in which neural-tube-derived Wnt protein activates Noggin expression in the medial dermo-myotome which in turn antagonizes the effect of lateral-plate-derived BMP4. In addition, we find that ectopic SHH expression also antagonizes the somite patterning activity of BMP4, and that this effect could be accounted for by Noggin activation laterally. Our results suggest that Noggin acts downstream of the Wnt and SHH pathways to specify the medial somitic compartment.

\section{MATERIALS AND METHODS}

\section{Microsurgical procedures}

Fertile eggs from chick embryos (JA57 strain from Institut de Sélection Animale, Lyon, France) were incubated at $37^{\circ} \mathrm{C}$ until they reached stage 12 (Hamburger and Hamilton, 1992). All surgical experiments were performed in ovo. Each experiment relies on at least 5 to 10 cases and up to 30 interpretable operated embryos for each gene were examined. The somite staging system developed by Ordahl (1993) has been used for numbering somites, i.e., the somite number in roman numeral corresponds to the number of the somite starting from the last segmented somite (somite I). Grafts of cell aggregates (see below) between the neural tube and the paraxial mesoderm or between the lateral plate and the paraxial mesoderm were performed as described in Pourquié et al. (1996). To perform neural tube ablations, bilateral incisions in the ectoderm were made along the segment to be removed (corresponding to a length of between 5 to 10 prospective somites). The segment was then removed using a micropipet. To graft QT6-SHH, CHO-Ng, Rat1-Wnt1 or the corresponding control cells, aggregates of cells were aspired into a micropipet and gently blown within the groove left after neural tube removal. Graft and ablation experiments were performed at the presomitic level and embryos were reincubated for a period of time ranging from 15 to 24 hours, which corresponds approximately to the formation of between 10 and 15 somites.

\section{Plasmids and retroviral vectors}

Probes for the avian Single-minded gene (Sim1), Pax-3, MyoD and BMP4 were described in Pourquié et al. (1996). The chick Noggin probe was produced from the plasmid described in Connolly et al. (1997) and was linearized using $X b a \mathrm{I}$ and transcribed with the T7 polymerase. SHH probe was produced as described in Riddle et al. (1993). Chick Wnt1 probe is described in Bally-Cuif and Wassef (1994). Pax-1 probe is produced from a $1.5 \mathrm{~kb}$ chicken Pax-1 cDNA encompassing the full-length coding region, cloned into pBKSII. The probe was linearized using $\mathrm{XbaI}$ and transcribed using T3 polymerase.

\section{In situ hybridization procedures and histology}

Whole-mount in situ hybridization procedures are as described in Henrique et al. (1995). Stained embryos were processed for sectioning using a Leica vibratome after albumin/gelatin inclusion. $50 \mu \mathrm{m}$ sections were collected on microscopic slides and mounted in Aquatex (Merck). Whole-mount stained embryos were photographed using a Wild M10 stereomicroscope (Leica). Sections were photographed with a Leica DM microscope using Nomarski optics.

\section{Cell lines}

CHO cells expressing Xenopus Noggin are a generous gift of Dr Richard Harland (UC Berkeley) and were described in Lamb et al. (1993). A stable QT6 cell line producing SHH/pBK was established as described in Duprez et al. (1996). Stable transfectants were selected in G418 and a single clone was isolated according to its high production of SHH and its ability to induce digit duplication in vivo. Wnt1-expressing Rat1 cells are a gift of Dr Roel Nusse. Production of the QT6-BMP4 cells has been described in Pourquié et al. (1996). In order to graft cell aggregates, confluent cultures were transfered to bacterial grade Petri dishes. After 24 or 48 hours, the cells formed compact aggregates, which were used for grafting in the embryo. 


\section{RESULTS}

\section{Distribution of Noggin during somitogenesis correlates with a role as a BMP4 antagonist}

Our previous observations prompted us to search for factors that might antagonize BMP4 during somite development (Pourquié et al., 1996). Xenopus Noggin was reported to have this activity and to be expressed in the developing neural tube (Smith and
Harland, 1992; Zimmerman et al., 1996). The avian homologue of Noggin has been cloned and its expression was examined during early stages (up to stage $7 \mathrm{HH}$ ) (Connolly et al., 1997). We examined chick Noggin expression by in situ hybridization during later stages when the somites become patterned (stage 10-21 HH). In addition, we compared the Noggin expression pattern with that of BMP4 and of the medial and lateral somite markers MyoD and Sim1, respectively (Fig. 1A-F).
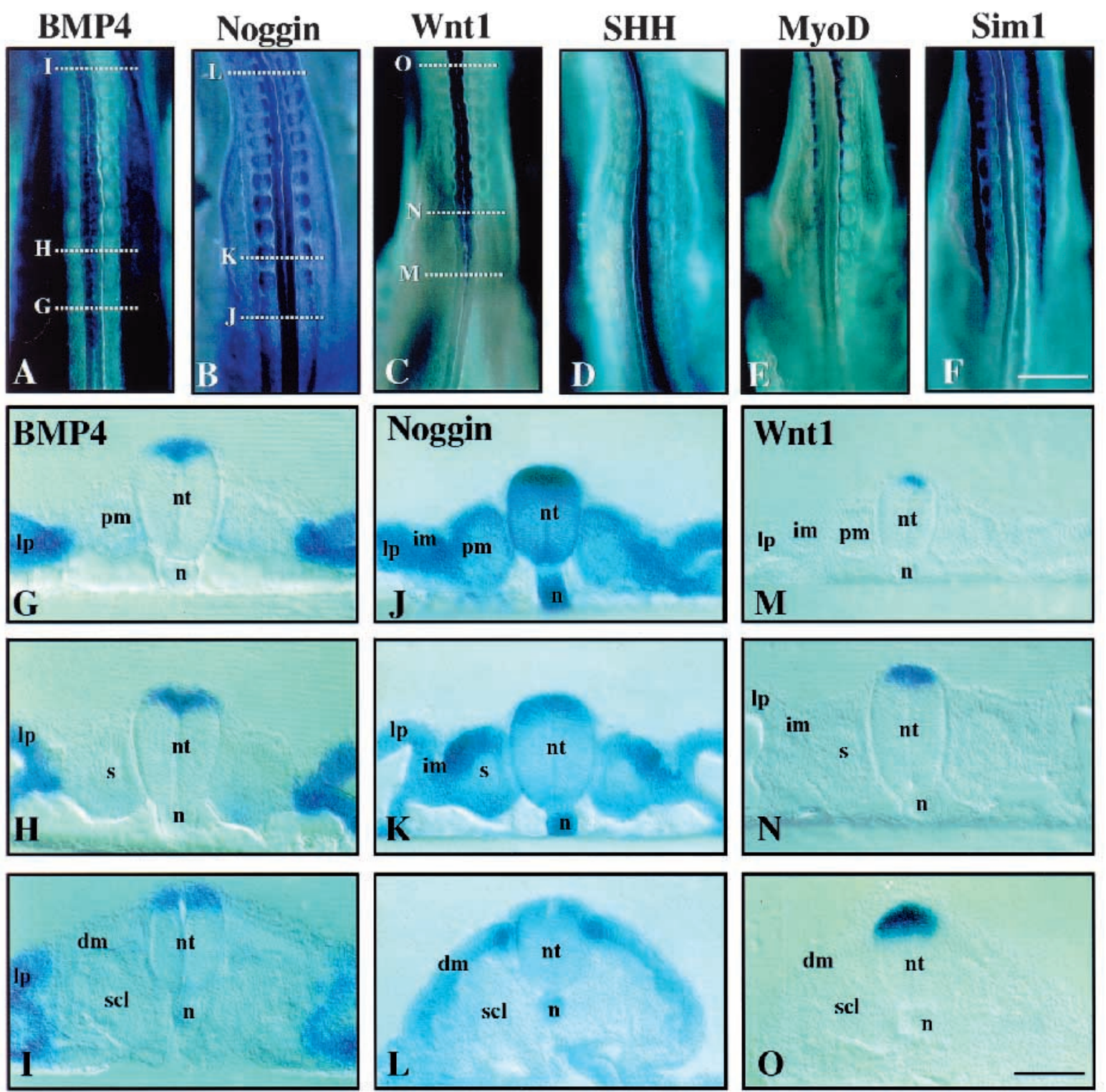

Fig. 1. Noggin expression in the developing mesoderm is consistent with a role in antagonizing lateral-plate-derived BMP4. (A-F) Wholemount in situ hybridizations of 20-somite chick embryos hybridized with BMP4 (A), Noggin (B), Wnt1 (C), SHH (D), MyoD (E) and Sim1 (F). Only the transition region between presomitic and segmented paraxial mesoderm is shown. Bar, $300 \mu \mathrm{m}$. (G-I) Transverse vibratome sections of the embryo shown in A. The A/P level of the sections is indicated in A. At the levels of presomitic mesoderm (G), of epithelial somite $(\mathrm{H})$ and of somite X (I), BMP4 is expressed in the lateral plate and the dorsal neural tube. (J-L) Transverse vibratome sections of the embryo shown in B. The A/P level of the sections is indicated in B. (J) At the level of the presomitic mesoderm, Noggin is detected in the intermediate mesoderm and lateral plate, the notochord, the floor plate and the dorsal neural tube. (K) At the level of the epithelial somite, Noggin is detected in the dorsolateral somite, the notochord and the dorsal neural tube. (L) At the level of somite X, Noggin is essentially expressed in the dorsomedial dermomyotome at the level of the dorsal lip. Expression in the dorsal neural tube and the notochord is downregulated. (M-O) Transverse vibratome sections of the embryo shown in C. The A/P level of the sections is indicated in C. At all three levels, Wnt1 is expressed in the dorsal neural tube in a domain similar to the BMP4-expressing domain. dm, dermomyotome; im, intermediate mesoderm; lp, lateral plate; n, notochord; nt, neural tube; pm, presomitic mesoderm; s, somite; scl, sclerotome. (Bar, $100 \mu \mathrm{m})$. 
At all stages examined, Noggin is detected in a broad caudal domain extending around the tail bud where it partly overlaps with the caudal BMP4 expression domain (Fig. 1A,B,G,J and data not shown). Throughout somitogenesis, Noggin expression is strong in the caudal neural tube, up to the level of the last formed somites (Fig. 1B,J). In this structure, Noggin is found in the dorsal BMP4-expressing region but is also transiently detected in the floor plate (Fig. 1G,J). BMP4 expression is maintained all along the rostral neural tube whereas Noggin becomes downregulated in the segmented region (Fig. $1 \mathrm{H}, \mathrm{K}, \mathrm{I}, \mathrm{L})$. In the notochord, Noggin is expressed up to the level of somite X (Fig. 1J,K,L) but downregulated opposite older somites.

In the caudal mesoderm, Noggin is detected at a low level in the lateral plate and at a higher level between the lateral plate and the presomitic paraxial mesoderm (Fig. 1B,J). This corresponds to the region of the prospective intermediate mesoderm, which also expresses BMP4 (Fig. 1A,G).

At the level of the newly formed somites, the expression profiles of Noggin and BMP4 become exclusive (Fig. $1 \mathrm{H}, \mathrm{K}, \mathrm{I}, \mathrm{L})$. In the transition region between the presomitic mesoderm and the early somites, Noggin is expressed according to a highly dynamic pattern (Fig. 1B). Transcripts are found in the lateral part of the rostral presomitic mesoderm and in the lateral part of somite I to III (Fig. 1K). This expression domain progressively moves medially starting with somite III as the somite becomes patterned along the mediolateral and dorsoventral axes. Noggin expression finally stabilizes in the dorsomedial dermomyotome from somite VI onwards (Fig. 1L). BMP4 expression in the mesoderm is restricted to the lateral plate at the corresponding stages (Fig. 1G-I). In stage-matched embryos, MyoD was detected medially and Sim1 laterally starting at the level of somite II-III. This represents the first indication of mediolateral polarity (Fig. 1E,F). In older embryos (stage 16 to 21), Noggin remains expressed in the same caudal structures but becomes progressively downregulated in the somites, so that by stage 22 , somitic expression is barely detectable (data not shown).

Therefore during avian somitogenesis, the Noggin and BMP4 expression patterns do not exhibit the simple mutually exclusive profiles observed during mesoderm patterning in Xenopus. However, as the somites become polarized along the mediolateral axis, Noggin expression becomes progressively localized to the medial somite, while BMP4 remains confined to the lateral plate. Thus the spatiotemporal expression profile of Noggin indicates a potential role as an antagonist of BMP4 in somite mediolateral patterning of the somite.

\section{Noggin can antagonize lateral somite specification}

To test for a potential role of Noggin in mediolateral patterning of the somite, we examined whether Noggin-expressing cells can antagonize lateral plate signaling in vivo. To this end, we grafted aggregates of control $\mathrm{CHO}$ cells, and $\mathrm{CHO}$ cells expressing Xenopus Noggin (CHO-Ng), between the lateral plate and the lateral somite at the level of the presomitic plate (Fig. 2A,D). Grafts of CHO-Ng cells resulted in the downregulation of expression of lateral markers such as Sim1 (Fig. 2F) and Pax-3 (data not shown) and in upregulation of the medial marker MyoD in the lateral somite (Fig. 2E). In contrast, grafts of control $\mathrm{CHO}$ cells had no effect on MyoD and Sim1 expression in the somite (Fig. 2A-C). These results suggest that secreted Noggin can block lateral-plate-derived

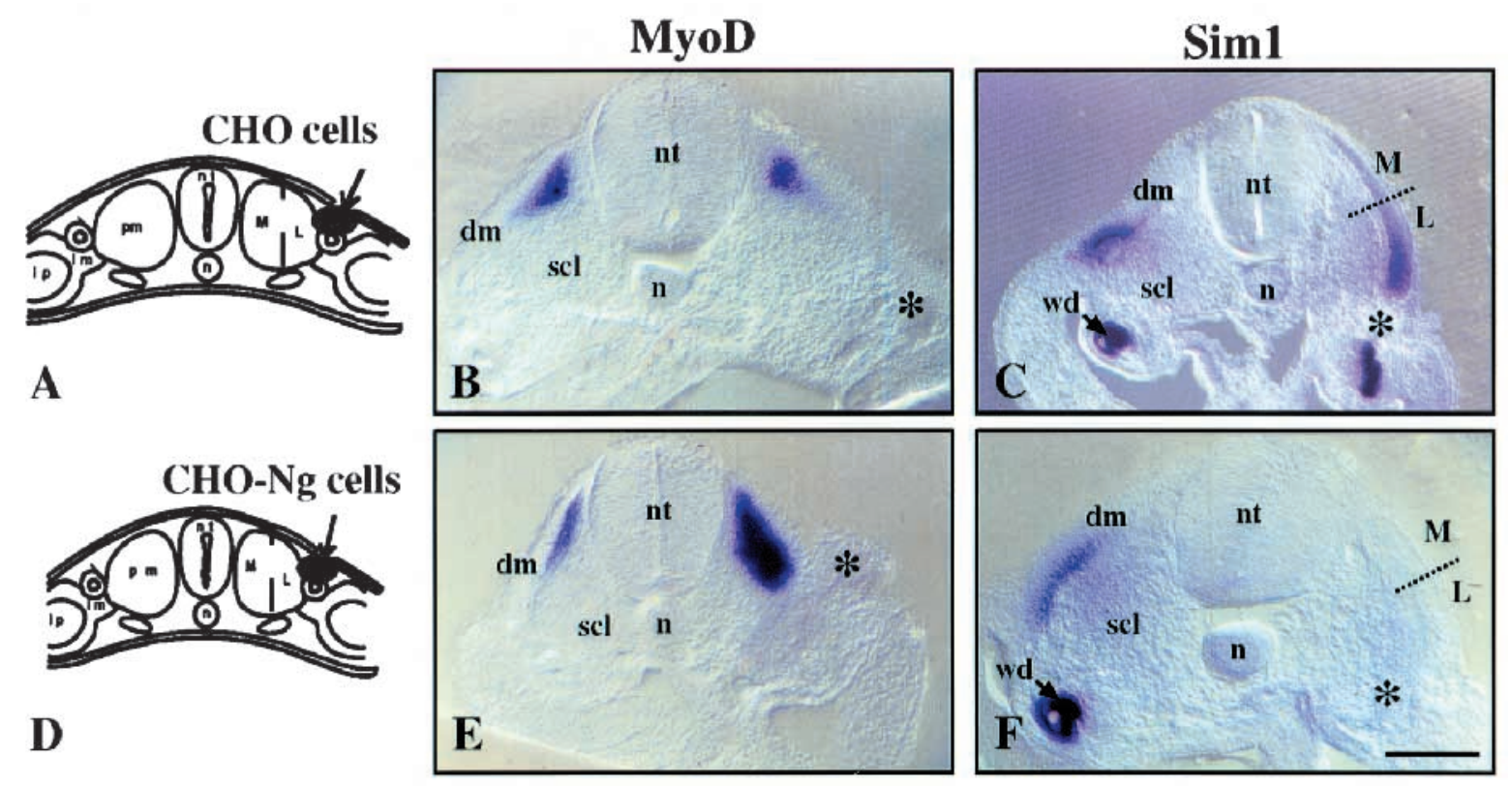

Fig. 2. Noggin-expressing cells can block lateral plate signaling. (A,D) Schematic transverse sections of E2 chick embryos illustrating the surgical operations. CHO (A) or Xenopus Noggin-expressing CHO cells (CHO-Ng) (D) are grafted between the lateral plate and the presomitic mesoderm. (B,C,E,F) Transverse vibratome sections of E3 chick embryos operated as described in A,D and hybridized as whole mounts with the MyoD (B,E) and Sim1 (C,F) probes. Grafted cells are indicated with an asterisk. Dotted lines demarcate the putative limit between medial (M) and lateral (L) somitic domains. (B, C) Graft of CHO cells has no effect on MyoD (B) expression nor on Sim1 expression (C). (E,F) Graft of CHO-Ng cells strongly upregulates the expression of the medial marker MyoD (E) normally repressed by lateral-plate-derived BMP4. These grafts also downregulate expression of the Sim1 lateral marker $(\mathrm{F})$ whose expression is controlled by lateral plate signals. dm, dermomyotome; nt, neural tube; n, notochord; scl, sclerotome; wd, Wolffian duct. (Bar, $110 \mu \mathrm{m})$. 
BMP4 signaling, resulting in the lateral somite acquiring a medial somite identity.

We have previously shown that ablation of the neural tube causes medial somitic cells to lose their medial characteristics and to acquire a lateral identity (Pourquié et al., 1996). We asked whether this manipulation affects Noggin expression. Ablation of the neural tube at the presomitic level leads to a downregulation of Noggin expression in the medial somite at the operation level whereas expression is maintained anteriorly and posteriorly to the zone of ablation (data not shown). Since Noggin is expressed in the neural tube prior to being detected in the somite, one could postulate the existence of an autoregulatory loop in which neural-tube-derived Noggin activates Noggin expression in the somite. To address this issue, we tested whether the implantation of Nogginexpressing cells after neural tube ablation could rescue Noggin expression in the somite. The neural tube was ablated over a distance of 5 to 10 somites at the presomitic level and the groove was filled with aggregates of Noggin-expressing or control cells (Fig. 3A,E). In both cases, Noggin somitic expression was not detected in the somites adjacent to the graft (Fig. 3B,F). These data indicate that the neural tube produces a signal, other than Noggin itself, which is required for Noggin expression in the medial somite.

We then asked whether grafting $\mathrm{CHO}-\mathrm{Ng}$ cells in place of the neural tube would rescue medial somite pattern thereby functionally replacing the endogenous Noggin somitic expression. As expected, in embryos in which the neural tube was substituted by control $\mathrm{CHO}$ cells, MyoD expression was downregulated and Sim1 expression extended into the medial somitic domain (Fig. 3C,D). By contrast, when $\mathrm{CHO}-\mathrm{Ng}$ cells were implanted, expression of MyoD was rescued and that of Sim1 downregulated in the medial somitic domains adjacent to the grafted cells (Fig. 3G,H). Therefore, these experiments suggest that Xenopus Noggin produced by the $\mathrm{CHO}$ cells can substitute for Noggin produced by the medial somite and therefore restore medial somitic identity.

Thus our data indicate that Noggin produced by the medial somite plays an important role in medial somite patterning potentially by blocking the BMP4 signal arising from the lateral plate. In addition, we demonstrate the existence of a signal different from Noggin that is produced by the neural tube and required for Noggin expression in the medial somite.

\section{Wnt1 activates Noggin expression in the medial somite and rescues medial somite patterning}

Several members of the Wnt family are expressed by the neural tube and in vitro experiments have demonstrated a role for Wnt1 in promoting somite myogenesis (Münsterberg et al., 1995; Stern et al., 1995). Wnt1 is expressed in the dorsal neural tube from the level of the first formed somites, which start to express Noggin at the same time (Fig. 1C,M,N,O). This correlation in expression profiles suggests that Wnt 1 could play a role in the regulation of Noggin in the somite.

To test for a role of Wnt1 in the activation of Noggin expression and in medial somite patterning, aggregates of

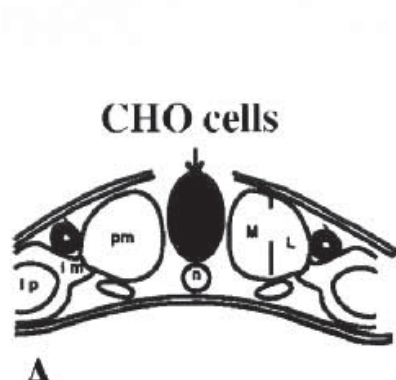

A

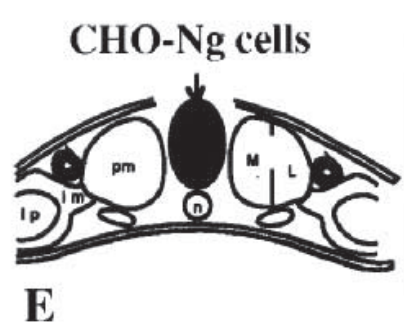

Noggin
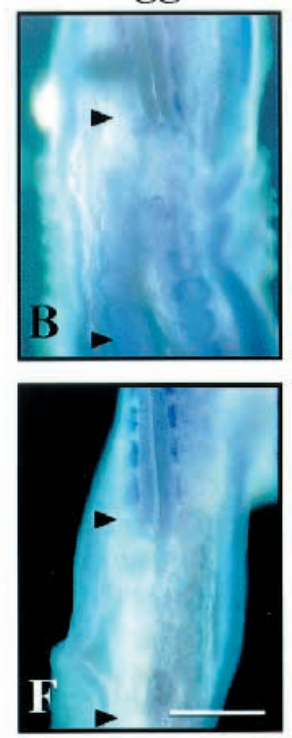

MyoD
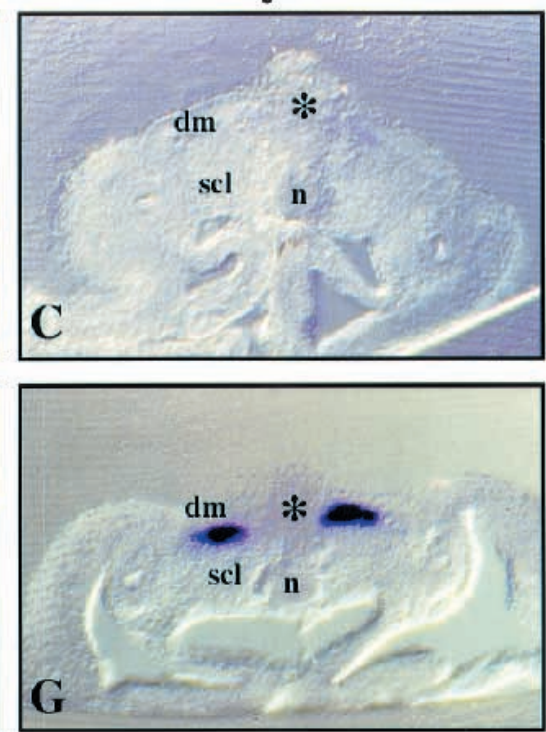

Sim1
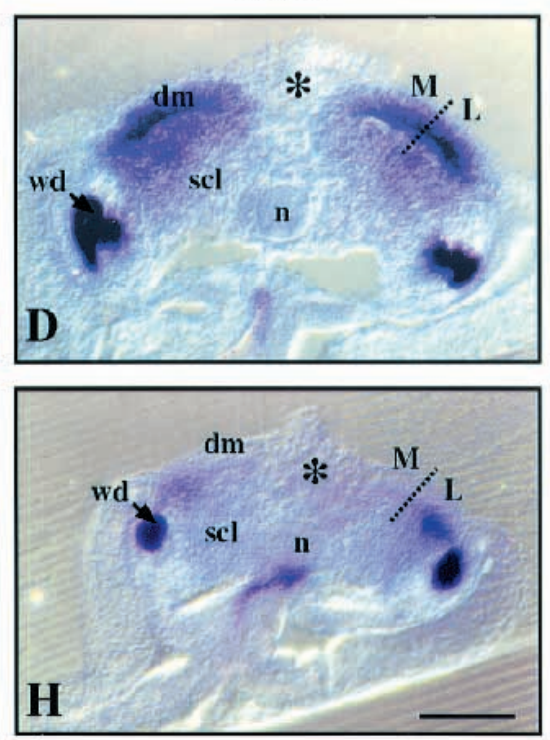

Fig. 3. Noggin-expressing cells can rescue medial somite patterning in embryos deprived of the neural tube. (A,E) Schematic transverse sections of E2 chick embryos illustrating the surgical operations. The neural tube is removed over a length corresponding to 5-10 somites at the presomitic level and replaced by aggregates of CHO (A) or CHO-Ng cells (E). (B,F) Whole-mount E3 embryos operated as described in A,E and hybridized with the Noggin probe. Only the operated region is shown. Whether the neural tube is replaced by $\mathrm{CHO}$ cells (B) or by $\mathrm{CHO}-$ $\mathrm{Ng}$ cells $(\mathrm{F})$, the Noggin-expressing domain in the medial somite disappears following the operation. Ablation extends between arrowheads. (Bar, $300 \mu \mathrm{m})$. (C,D,G,H) Transverse vibratome sections of E3 chick embryos operated as described in A,E and hybridized as whole mounts with the MyoD $(\mathrm{C}, \mathrm{G})$ and Sim1 probe $(\mathrm{D}, \mathrm{H})$. Grafted cells are indicated by an asterisk. Dotted lines demarcate the putative limit between medial (M) and lateral (L) somitic domains. (C,D) Removal of the neural tube and graft of CHO cells is followed by a loss of MyoD expression (C) whereas Sim1 expression spreads in the medial dermomyotome (D). (G,H) When the neural tube is replaced by CHO-Ng cells, MyoD expression in the medial somite is rescued $(\mathrm{G})$ while $\mathrm{Sim} 1$ is normally confined to the lateral somite $(\mathrm{H})$. Therefore Noggin is able to rescue medial somite patterning. dm, dermomyotome; n, notochord; nt, neural tube; scl, sclerotome; wd, Wolffian duct. Bar, $90 \mu \mathrm{m}$. 

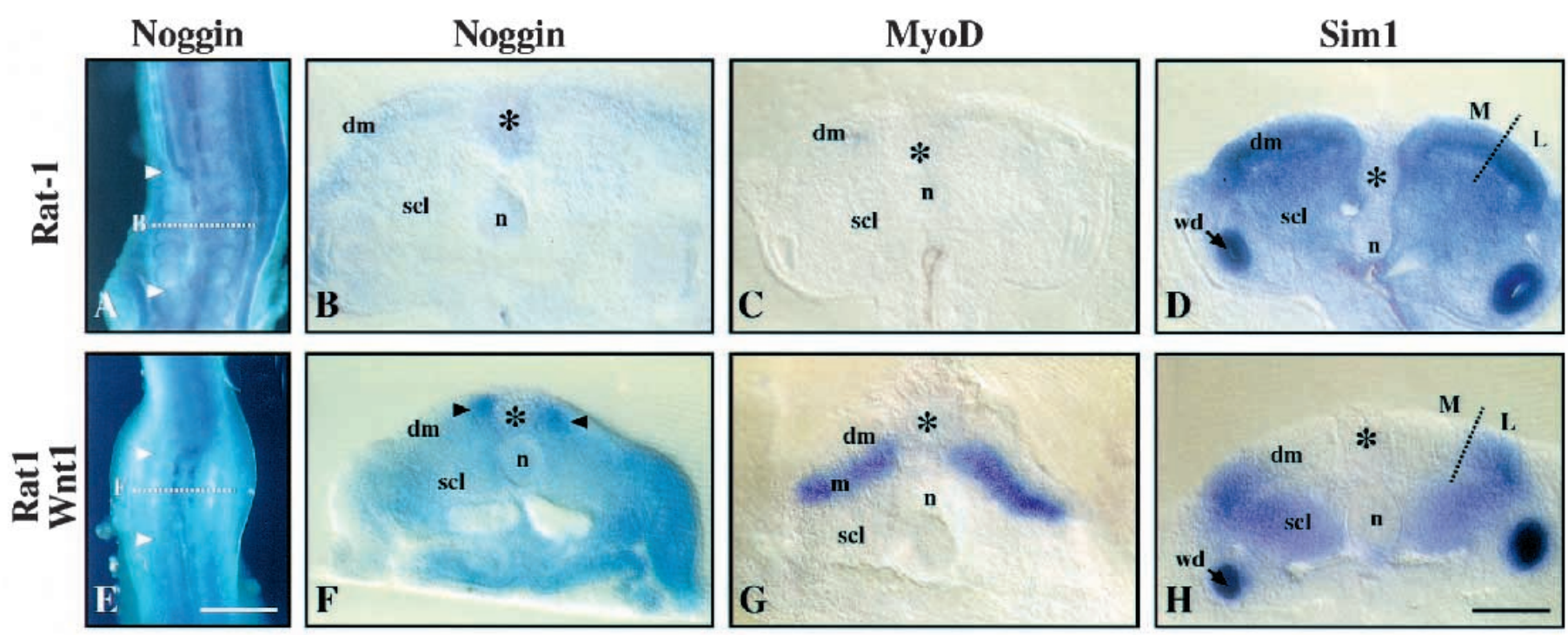

Fig. 4. Wnt1 can rescue Noggin expression in the somite and consequently medial somite patterning. (A,E) E3 chick embryos hybridized as whole mounts with the Noggin probe after substitution of the neural tube by Rat1 (A) or Wnt1-expressing Rat1 (Rat1-Wnt1) cells (E) as described in Fig. 3(A,E). Ablation extends between arrowheads. (Bar=300 $\mu \mathrm{m}$ ). (B,F) Transverse vibratome sections through the embryos shown in A,E at the level of the dotted lines. As shown in Fig. 3, Noggin expression disappears after substitution of the neural tube by Rat1 cells $(A, B)$. On the contrary, rescue of Noggin expression is observed in a narrow domain adjacent to the graft when Rat1-Wnt1 cells are grafted (E, arrowheads in F). (C,D,G,H) Transverse vibratome sections of E3 chick embryos operated as described in A,E and hybridized in whole-mount with the MyoD (C,G) and Sim1 (D,H) probes. Grafted cells are indicated by an asterisk. (C,D) As shown in Fig. 3, MyoD (C) expression disappear from the dermomyotome while Sim1 (D) expression extends in the medial somite after substitution of the neural tube by Rat1 cells. $(\mathrm{G}, \mathrm{H})$ Neural tube replacement by Rat1-Wnt1 cells rescues normal MyoD expression (G) and restores Sim1 expression (H) in the lateral somite. dm, dermomyotome; m, myotome; n, notochord; nt, neural tube; scl, sclerotome; wd, Wolffian duct. Bar, $100 \mu \mathrm{m}$.

control Rat1 or of Wnt1-expressing Rat1 (Rat1-Wnt1) cells were substituted for the neural tube at the presomitic level as described in Fig. 3. As shown for the control CHO cells in the previous section, grafts of control Rat1 cells led to the loss of Noggin expression in the medial somite at the level of the operation (Fig. 4A,B). In contrast, transplantation of Rat1Wnt1 cells rescued Noggin expression in the medial somite in a narrow domain adjacent to the implanted Wnt1-producing cells (Fig. 4E,F). Moreover, while Rat1 control cells, like CHO cells, were not able to rescue the medial somitic pattern (compare Figs 4C,D and 3C,D), Wnt1-expressing cells rescued the medial somite pattern as evidenced by strong MyoD expression and Sim1 downregulation in the medial domain of somites from operated embryos (Fig. 4G,H).

Interestingly, the rescue of Noggin expression occurs only in cells in very close proximity to the Wnt1-expressing cells (Fig. 4E,F), whereas Sim1 is downregulated over a longer distance corresponding approximately to 20 cell diameters (Fig. 4H). This suggests two possibilities: either lower concentrations of Wnt1 protein are required to downregulate Sim1 expression than to activate Noggin, indicating that Wnt1 acts as a morphogen; or Wnt1 acts at short range to induce Noggin expression which in turn diffuses over a long range to downregulate Sim1. The latter possibility is more likely since

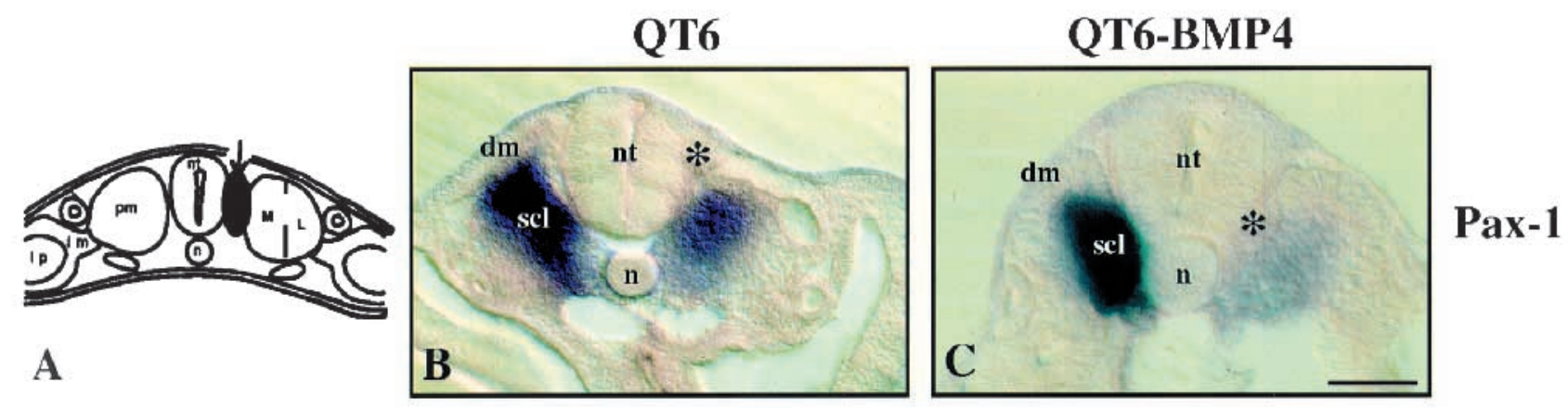

Fig. 5. BMP4 can interfere with the SHH signaling pathway. (A) Schematic transverse section of E2 chick embryos illustrating the graft of QT6 or BMP4-expressing QT6 (QT6-BMP4) cells (in black) between the neural tube and the presomitic mesoderm. (B,C) Transverse vibratome sections of E3 chick embryos operated as described in A and hybridized as whole mounts with a Pax1 probe. Grafted cells are indicated by an asterisk. Graft of QT6 cells in this location only slightly affects Pax1 expression in the operated side (B) while graft of QT6-BMP4 cells induces a strong downregulation of Pax 1 expression (C). Note that sclerotome formation is not affected. dm, dermomyotome; m, myotome; $\mathrm{n}$, notochord; nt, neural tube; scl, sclerotome; wd, Wolffian duct. Bar, $100 \mu \mathrm{m}$. 
Noggin is known to block BMP4 activity which is required for Sim1 expression (Zimmerman et al., 1996; Pourquié et al., 1996). The fact that grafts of both Noggin- and Wnt1-expressing cells can rescue medial somite patterning is in agreement with a relay system involving Noggin activation by Wnt 1 .

\section{Evidence for opposing actions of SHH and BMP4 in somite patterning involving Noggin activation}

Dorsoventral patterning of the neural tube is established through the opposing actions of BMP4 produced dorsally and of SHH produced ventrally (Liem et al., 1995). We have tested whether such an antagonism exists at the somite level in which BMP4 is produced laterally and SHH medially. To that end, we examined whether BMP4 interferes with the positive regulation of the sclerotomal marker Pax-1 by SHH (Fan and TessierLavigne, 1994; Chiang et al., 1996). Indeed, grafts of BMP4expressing QT6 cells placed between the neural tube and the paraxial mesoderm led to a strong downregulation of Pax-1 expression in the sclerotome (Fig. 5A-C).

We subsequently examined whether $\mathrm{SHH}$ also plays a role in mediolateral patterning of the somite. QT6 cells overexpressing chick full-length SHH (QT6-SHH) were grafted lateral to the somite, to test for their ability to interfere with somite lateralization (Fig. 6A). As observed for the grafts of Noggin-expressing cells, QT6-SHH cells induced a strong upregulation of the medial marker MyoD and a downregu-
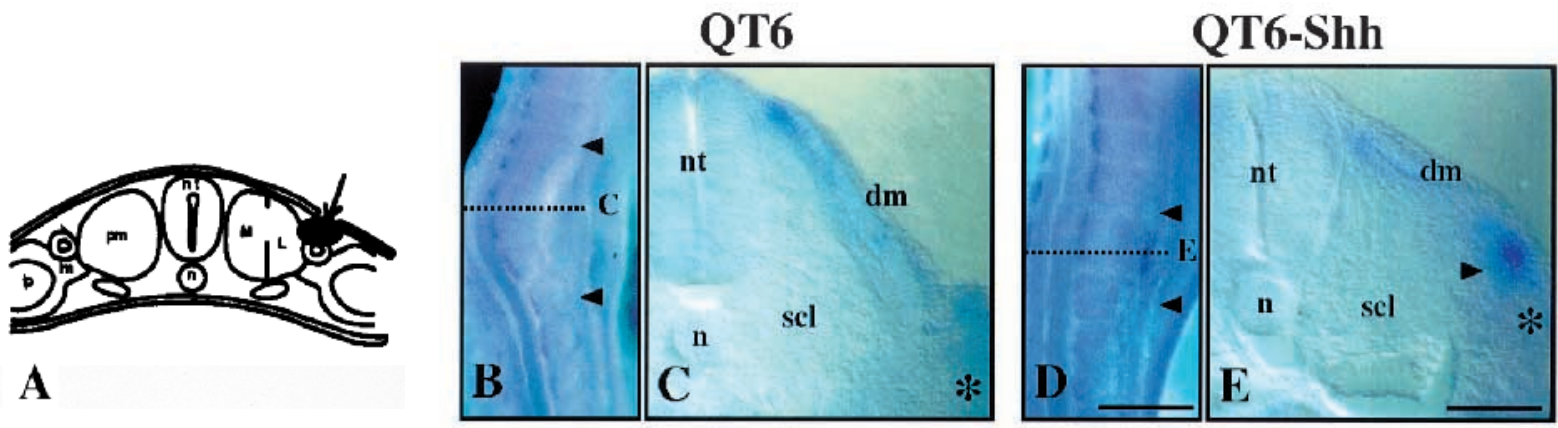

Noggin

Fig. 6. Sonic Hedgehog can activate ectopic Noggin expression and antagonize BMP4 effects on the lateral somite. (A) Schematic transverse sections of E2 chick embryo illustrating the graft of QT6 or SHH-expressing QT6 (QT6$\mathrm{SHH}$ ) cells (in black) between the lateral plate and the presomitic mesoderm.

$(\mathrm{B}, \mathrm{D})$ Whole-mount in situ hybridization using a Noggin probe on E3 chick embryos grafted as described in A with QT6 (B) or QT6-SHH cells (D). Limits of the graft are indicated by arrowheads. (Bar, $300 \mu \mathrm{m}$ ). (C,E) Transverse vibratome sections corresponding to the embryos shown in B,D, respectively. Grafted cells are indicated by an asterisk. The grafts of QT6-SHH cells induce ectopic Noggin expression in cells of the lateral somite adjacent to the grafted aggregate (D, arrowhead in E). No such effect is observed when QT6 cells are grafted $(B, C)$.

(F-K) Transverse vibratome sections of E3 chick embryos
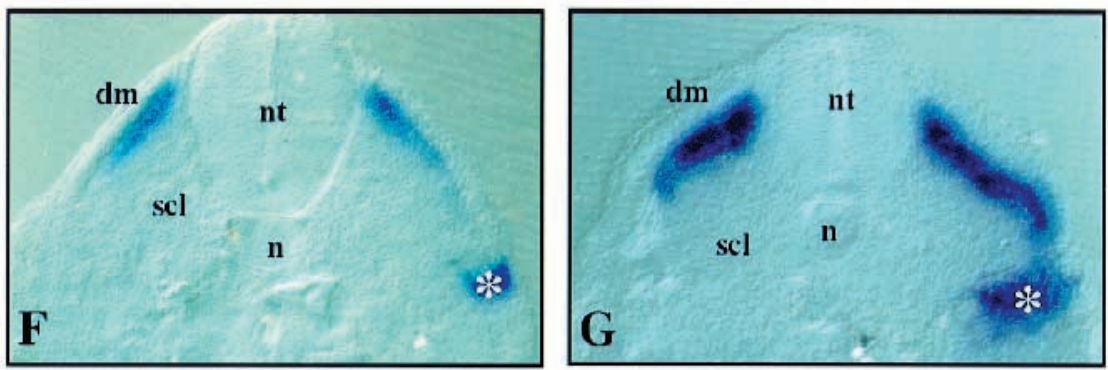

\section{MyoD}
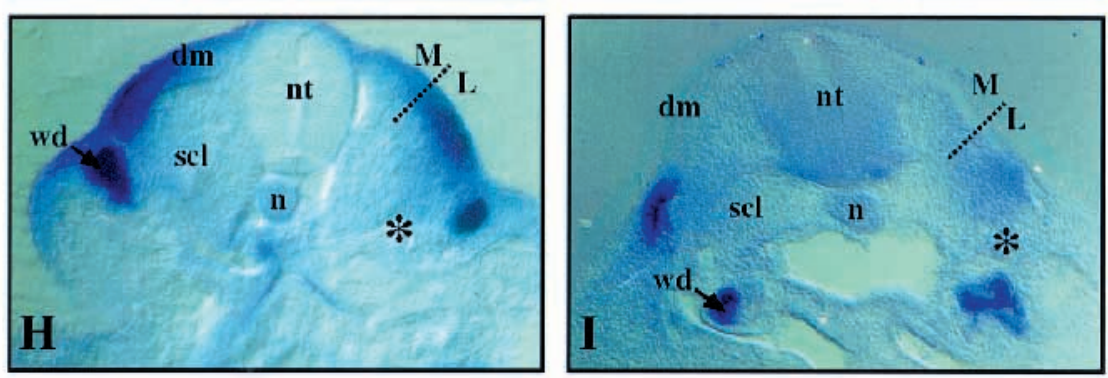

Sim 1
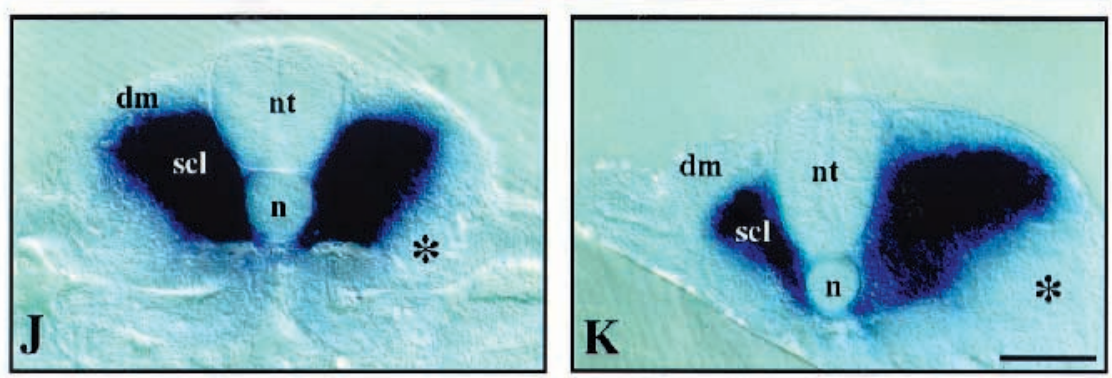

Pax-1 operated as described in A and hybridized as whole mounts with the MyoD (F,G), the Sim1 (H,I) and the Pax1 (J,K) probes. (F-I) Grafts of QT6-SHH cells can upregulate MyoD expression (G) and downregulate Sim1 expression (I) in the lateral somite in a way similar to Noggin-expressing cells. Graft of control QT6 cells has no such effect on MyoD (F) and Sim1 (H) expression. Note that the grafted QT6 cells express MyoD (F, G). (J,K) Grafts of QT6-SHH cells induce a strong upregulation of Pax1 in the lateral somite and an hypertrophy of the paraxial mesoderm on the operated side (K). Such an effect is not observed with the graft of QT6 cells (J). dm, dermomyotome; n, notochord; nt, neural tube; scl, sclerotome; wd, Wolffian duct. Bar, $100 \mu \mathrm{m}$. 
lation of Sim1 (Fig. 6G,I). Expression of MyoD and Sim1 was not affected by grafting control QT6 cells (Fig. 6F,H). Grafting QT6-SHH cells, but not control QT6 cells, produced a strong upregulation of the ventral marker Pax1 without disrupting the dermomyotome structure dorsally (Fig. 6J,K). Such an effect could potentially be mediated by the activation of Noggin. Indeed, SHH-expressing cells, but not control QT6 cell grafts, induced the ectopic expression of Noggin in the lateral dermomyotome compartment (Fig. 6D,E). Thus SHH appears to be involved in mediolateral somite patterning and this may proceed through Noggin activation.

It is noteworthy that the operated side appears wider than the control one following grafts of SHH-expressing cells (Fig. $6 \mathrm{~K})$. This hypertrophy of the paraxial mesoderm was not detected with Noggin-expressing $\mathrm{CHO}$ grafts. It suggests an effect of SHH on cell proliferation, which is in agreement with its proliferative effect on the sclerotome demonstrated in vitro (Fan et al., 1995). Therefore, our experiments demonstrate that Noggin is downstream of the SHH pathway, although it is unlikely to be the sole gene to be activated in response to SHH.

\section{DISCUSSION}

We have analyzed the properties of molecules likely to play a role in opposing lateral somite specification by BMP4. We report that the BMP4 antagonist Noggin is expressed in the developing medial somite in a manner compatible with a role in this process. We demonstrate that Noggin-expressing cells are able to block lateral plate signaling and that the implantation of these cells in place of the neural tube rescues medial somite patterning as indicated by activation of $\mathrm{MyoD}$ expression and the downregulation of Sim1. In addition, we demonstrate that Noggin expression in the somite is under the control of a neural-tube-derived factor. Wnt1, which is expressed in the dorsal neural tube, can rescue the somitic Noggin expression. We show that SHH, produced by the notochord and the floor plate, can block lateral plate signaling and that this effect may result from local ectopic activation of Noggin. Therefore, we propose that Noggin is a key player in promoting medial somite patterning in vivo and does so by antagonizing lateral-plate-derived BMP4. In addition, our data support a model in which Noggin activation lies downstream of the SHH and Wnt signaling pathways. Somite medial patterning would be the result of a signaling cascade in which SHH and Wnt1 produced by the axial organs promote Noggin expression in the medial somite and in the floor plate and notochord which in turn antagonize lateral-plate-derived BMP4 (Fig. 7).

\section{The BMP4 antagonizing activity of Noggin results in mediolateral patterning of the avian somite}

Evidence for the role of lateral-plate-derived BMP4 in somite specification comes from the ability of BMP4-producing cells to convert the medial somite to a lateral fate, as evidenced by the expression patterns of the Sim 1 and MyoD genes (Pourquié et al., 1996; Tonegawa et al., 1997). Here, we provide additional evidence for the role of the BMP4 signaling pathway in lateral somite specification. We show that the BMP4 antagonist Noggin is able to block lateral plate signaling since grafts of Noggin-expressing cells are able to convert the lateral

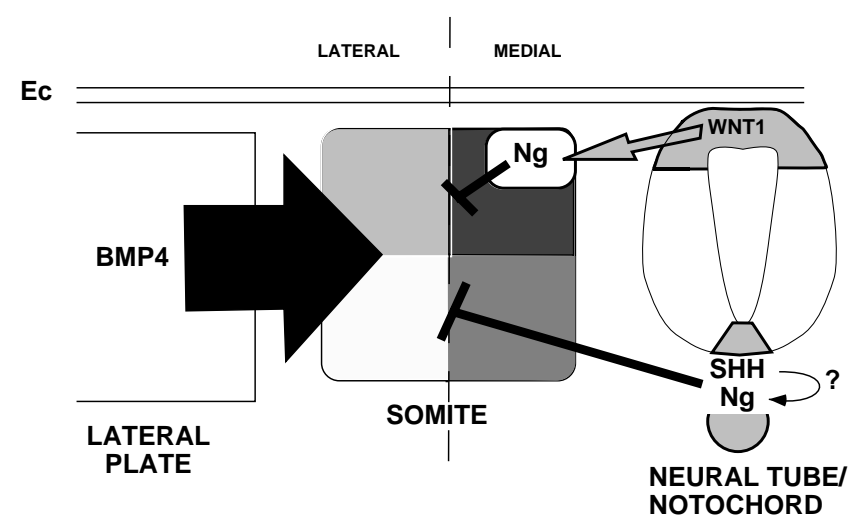

Fig. 7. Schematic representation of the interactions between axial and lateral signaling molecules during somite mediolateral patterning. Lateral-plate-derived BMP4 specifies the lateral somitic compartment resulting in Sim1 activation and the inhibition of the onset of myogenesis. The BMP4 effect is antagonized by dorsal and ventral axial factors, which include $\mathrm{SHH}$ and Wnt1. These axial factors repress Sim 1 activation and promote the activation of Pax 1 ventrally and MyoD dorsally. Noggin appears to be activated by and to act downstream of SHH and Wnt1: Wnt1 activates Noggin expression in the medial dermomyotome and SHH is able to antagonize BMP4 ventrally, potentially by activating Noggin expression in the notochord and floor plate. Noggin is likely to directly mediate the opposing effect of Wnt1 and SHH to BMP4. The action of these axial factors ultimately results in determination of the epaxial muscles dorsally and of the vertebral column ventrally while the hypaxial muscle lineage is specified by lateral-plate-derived factors. Ec, ectoderm.

somite to a medial fate as shown by Sim1 downregulation and MyoD expression. In addition, the medial somitic expression domain of Noggin is compatible with a role in neutralizing the lateral BMP4 signal.

The Noggin and BMP4 expression patterns that we describe do not exhibit the simple complementarity observed during Xenopus gastrulation. In the neural tube, Noggin is principally detected in the roof plate of the caudal region where its expression overlaps with that of BMP4. Although we have no evidence that Noggin and BMP4 are expressed in exactly the same cells, they are expressed in broadly the same territories. This situation suggests a novel mode for Noggin action in which it would directly act in a BMP4-expressing territory. Noggin expression in the neural tube is downregulated at the level of the first formed somites whereas BMP4 expression extends more rostrally. This expression pattern could provide a means of restricting BMP4 activity, important for dorsoventral neural tube patterning, to the segmented level of the embryo (Liem et al., 1995).

In the mesoderm of the chick embryo, Noggin tends to be expressed more axially whereas BMP4 is found laterally. In the caudal part of the embryo, at the level of the presomitic mesoderm, the domain of Noggin expression coincides with the medial border of the BMP4 expression domain in the lateral plate. This juxtaposition of expression domains might act as a barrier to protect the unpatterned paraxial mesoderm from BMP4 action. Recently, BMP4 was shown to be able to recruit paraxial mesoderm to a lateral plate fate which included the activation of BMP4 expression (Tonegawa et al., 1997). BMP4, 
like its fly homolog DPP, is thought to be able to autoactivate its own expression (Bier, 1997; Jones et al., 1996). Therefore, the role of such a barrier might be important to precisely demarcate the BMP4-expressing domain, since the caudal paraxial mesoderm is competent to respond to BMP4 by activating BMP4 itself.

Starting at the level of the newly formed somite, the expression profiles of Noggin and BMP4 in the paraxial mesoderm become progressively exclusive, culminating in Noggin expression in the medial somite and BMP4 in the lateral plate. At this level, Noggin might antagonize lateralplate-derived BMP4 from a distance thereby establishing an activity gradient as was described in Xenopus and fly gastrulation (Dosch et al., 1997; Tonegawa et al., 1997; Nellen et al., 1996; Lecuit et al., 1996). This type of Noggin-BMP4 interaction could result in appropriate BMP4 concentrations to allow medial and lateral patterning of the somite.

\section{Role of Noggin in the activation of myogenesis}

Our experiments demonstrate that Noggin plays an important role in promoting myogenesis. Noggin-expressing cells induce ectopic MyoD expression when grafted lateral to the somite and rescue endogenous MyoD expression when substituted for the neural tube. Interestingly, Noggin expression becomes restricted to the medial dermomyotome, where the committed myoblasts first arise. Later on, Noggin expression is closely associated with a structure called the dorsal lip of the dermomyotome which was recently shown to contribute the myoblasts of the early myotome (Denetclaw et al., 1997). A role in promoting muscle differentiation has also been ascribed to Noggin in Xenopus. In the frog, Noggin behaves as a bona fide dorsalizing factor of the Spemann organizer and is able to induce muscle differentiation from prospective ventral mesoderm normally fated to give rise to blood and mesenchyme (Smith et al., 1993). This role is thought to be mediated through its antagonizing effect on BMP4. Remarkably, MyoD and myf5 are expressed in the frog gastrula in territories that prefigure the prospective somitic field. Expression of these two genes has also been shown to be controlled by Noggin and BMP4 at the gastrula stage (Dosch et al., 1997). Our experiments indicate that, in the chick embryo, expression of the MyoD and myf5 genes is also controlled by the same BMP4/Noggin patterning system but at a much later stage of development, i.e. after somitogenesis. Future experiments will address more directly the role of Noggin in skeletal muscle cell differentiation.

\section{Wnt1 acts upstream of Noggin in the developing somite}

We show that Wnt1 is sufficient to maintain expression of Noggin in the medial somite. Wnt1-expressing cells can rescue Noggin expression in the somite and restore normal MyoD and Sim1 expression domains in embryos in which the neural tube has been ablated. The only other available evidence for Noggin acting downstream of the Wnt pathway derives from studies performed in Xenopus. During frog gastrulation, the Spemann organizer is known to be induced in response to signals involving members of the Wnt transduction pathway resulting in the activation of the Siamois transcription factor. This gene will, in turn, activate Noggin expression in the Organizer (Carnac et al., 1996), which subsequently acts on the adjacent mesodermal territory to promote MyoD activation and muscle differentiation.
In the chick embryo, several Wnt family members (Wnt1, Wnt3A and Wnt4) have been shown to regulate MyoD expression and promote myogenesis from the paraxial mesoderm (Münsterberg et al., 1995). Here, we provide additional evidence that Wnt proteins are involved in MyoD regulation and we show that the effect of Wnt molecules on MyoD activation and medial somite patterning is likely to be mediated by activating Noggin expression which then neutralizes BMP4 signaling. Interestingly, this is in good agreement with the hypothesis proposed several years ago in which muscle was proposed to be a default pathway of the developing paraxial mesoderm (Pourquié et al., 1993). This situation is analogous to that encountered in neural induction in the frog in which neural inducers have been essentially shown to antagonize BMP4 to promote expression of a default neural phenotype (Hemmati-Brivanlou and Melton, 1997).

\section{Role of Noggin in mediating SHH antagonism to BMP4}

We have observed that SHH-producing cells are able to mimick the effect of Noggin-producing cells in blocking lateral plate signaling. In this assay, SHH-expressing cells induced locally the ectopic expression of chick Noggin. It is likely that the inhibition of lateral plate signaling mediated by BMP4 resulted from the ectopic activation of Noggin. This suggests that in vivo SHH may act upstream of Noggin in some tissues. The ectopic activation of Noggin is, however, unexpected since ablation of the neural tube which results in the loss of Noggin expression, left a potential source of $\mathrm{SHH}$ signaling, i.e. the notochord. However, in embryos grafted with SHH-expressing cells, Noggin expression is only detected in very close proximity to the grafted cells. This observation suggests that Noggin activation occurs in response to high concentrations of $\mathrm{SHH}$. Therefore, the SHH concentration provided by the notochord might not be sufficient to rescue Noggin expression in embryos in which the neural tube is ablated. This argues against a direct regulation of Noggin expression in the somite by $\mathrm{SHH}$ signaling. However, in the caudal part of the embryo, SHH and Noggin are found to be coexpressed in the notochord and in the floor plate. In these structures, the transient Noggin expression may be regulated by $\mathrm{SHH}$ and might also play a role in mediolateral patterning of the adjacent ventral somite. Our data are in agreement with models recently proposed in which $\mathrm{SHH}$ and Wnt act synergistically to promote myogenesis (Münsterberg et al., 1995). We propose that Noggin acts as a downstream effector of these two signaling pathways important for the patterning of the medial somite (Fig. 7). It is important to note that such a role for Noggin is a much later-acting one than any role in primary dorsalisation wherein signals from the gastrula midline would neutralise ventralizing ones in establishing boundaries for paraxial mesoderm and neural plate. Such an earlier role was tested in chick and not found by Connolly et al. (1997) whose experiments with Noggin would not have been expected to perturb the later-acting role proposed here.

We thank Drs Mike Mc Grew, Patrick Lemaire, Christo Goridis, Chen-Ming Fan, Kim Dale and Malek Djabali for critical reading of this manuscript. We thank Dr Richard Harland for the generous gift of CHO cells expressing Xenopus Noggin and Dr Roel Nusse for the Rat1-Wnt1 cells. We are indebted to Drs Osamu Saitoh and Muthu Periasamy for the generous gift of the $M y o D$ probe, to Drs Rudi 
Balling and Haruhiko Koseki for the gift of the Pax-1 plasmid and to Marion Wassef for the Wnt1 plasmid. Financial support was provided by the Centre National de la Recherche Scientifique (CNRS), the Association Française contre les myopathies (AFM), the Association pour la Recherche contre le Cancer (ARC), and the Fondation pour la Recherche Médicale (FRM).

\section{REFERENCES}

Aoyama, H. and Asamoto, K. (1988). Determination of somite cells: independence of cell differentiation and morphogenesis. Development 104, $15-28$.

Bally-Cuif, L. and Wassef, M. (1994). Ectopic induction and reorganization of Wnt-1 expression in quail/chick chimeras. Development 120, 3379-3394.

Bier, E. (1997). Anti-neural inhibition: a conserved mechanism for neural inductions. Cell 89, 681-684.

Brand-Saberi, B., Ebensperger, C., Wilting, J., Balling, R. and Christ, B. (1993). The ventralizing effect of the notochord on somite differentiation in chick embryos. Anat. Embryol. (Berl). 188, 239-245.

Buffinger, N. and Stockdale, F. E. (1994). Myogenic specification in somites: induction by axial structures. Development 120, 1443-1452.

Carnac, G., Kodjabachian, L., Gurdon, J. B. and Lemaire, P. (1996). The homeobox gene Siamois is a target of the Wnt dorsalisation pathway and triggers organiser activity in the absence of mesoderm. Development $\mathbf{1 2 2}$, 3055-3065

Chiang, C., Litingtung, Y., Lee, E., Young, K. E., Corden, J. L., Westphal, H. and Beachy, P. A. (1996). Cyclopia and defective axial patterning in mice lacking Sonic hedgehog gene function. Nature 383, 407-413.

Christ, B., Brand-Saberi, B., Grim, M. and Wilting, J. (1992). Local signalling in dermomyotomal cell type specification. Anat. Embryol. (Berl). 186, 505-510.

Christ, B. and Ordahl, C. P. (1995). Early stages of chick somite development. Anat. Embryol. (Berl). 191, 381-396.

Connolly, D. J., Patel, K. and Cooke, J. (1997). Chick noggin is expressed in the organizer and neural plate during axial development, but offers no evidence of involvement in primary axis formation. Int. J. Dev. Biol. 41, 389396.

Cossu, G., Tajbakhsh, S. and Buckingham, M. (1996). How is myogenesis initiated in the embryo? Trends. Genet. 12, 218-223.

Denetclaw, W. F. J., Christ, B. and Ordahl, C. P. (1997). Location and growth of epaxial myotome precursor cells. Development 124, 1601-1610.

Dosch, R., Gawantka, V., Delius, H., Blumenstock, C. and Niehrs, C. (1997). Bmp-4 acts as a morphogen in dorsoventral mesoderm patterning in Xenopus. Development 124, 2325-2334.

Duprez, D., Bell, E. J., Richardson, M. K., Archer, C. W., Wolpert, L., Brickell, P. M. and Francis-West, P. H. (1996). Overexpression of BMP-2 and BMP-4 alters the size and shape of developing skeletal elements in the chick limb. Mech. Dev. 57, 145-157.

Echelard, Y., Epstein, D. J., St-Jacques, B., Shen, L., Mohler, J., McMahon, J. A. and McMahon, A. P. (1993). Sonic hedgehog, a member of a family of putative signaling molecules, is implicated in the regulation of CNS polarity. Cell 75, 1417-1430.

Fan, C. M. and Tessier-Lavigne, M. (1994). Patterning of mammalian somites by surface ectoderm and notochord: evidence for sclerotome induction by a hedgehog homolog. Cell 79, 1175-1186.

Fan, C. M., Porter, J. A., Chiang, C., Chang, D. T., Beachy, P. A. and Tessier-Lavigne, M. (1995). Long-range sclerotome induction by sonic hedgehog: direct role of the amino-terminal cleavage product and modulation by the cyclic AMP signaling pathway. Cell 81, 457-465.

Goulding, M., Lumsden, A. and Paquette, A. J. (1994). Regulation of Pax-3 expression in the dermomyotome and its role in muscle development. Development 120, 957-971.

Graff, J. M. (1997). Embryonic patterning: to BMP or not to BMP, that is the question. Cell 89, 171-174.

Hamburger, V. and Hamilton, H. L. (1992). A series of normal stages in the development of the chick embryo (1951). Dev. Dyn. 195, 231-272.

Hemmati-Brivanlou, A. and Melton, D. (1997). Vertebrate embryonic cells will become nerve cells unless told otherwise. Cell 88, 13-17.

Henrique, D., Adam, J., Myat, A., Chitnis, A., Lewis, J. and Ish-Horowicz, D. (1995). Expression of a Delta homologue in prospective neurons in the chick. Nature 375, 787-790.

Hogan, B. L. (1996). Bone morphogenetic proteins: multifunctional regulators of vertebrate development. Genes Dev. 10, 1580-1594.
Hollyday, M., McMahon, J. A. and McMahon, A. P. (1995). Wnt expression patterns in chick embryo nervous system. Mech. Dev. 52, 9-25.

Jones, C. M., Armes, N. and Smith, J. C. (1996). Signalling by TGF-beta family members: short-range effects of Xnr-2 and BMP-4 contrast with the long-range effects of activin. Curr. Biol. 6, 1468-1475.

Krauss, S., Concordet, J. P. and Ingham, P. W. (1993). A functionally conserved homolog of the Drosophila segment polarity gene hh is expressed in tissues with polarizing activity in zebrafish embryos. Cell 75, 1431-1444.

Lamb, T. M., Knecht, A. K., Smith, W. C., Stachel, S. E., Economides, A. N., Stahl, N., Yancopolous, G. D. and Harland, R. M. (1993). Neural induction by the secreted polypeptide noggin. Science 262, 713-718.

Lecuit, T., Brook, W. J., Ng, M., Calleja, M., Sun, H. and Cohen, S. M. (1996). Two distinct mechanisms for long-range patterning by Decapentaplegic in the Drosophila wing. Nature 381, 387-393.

Liem, K. F. J., Tremml, G., Roelink, H. and Jessell, T. M. (1995). Dorsal differentiation of neural plate cells induced by BMP-mediated signals from epidermal ectoderm. Cell 82, 969-979.

Maroto, M., Reshef, R., Münsterberg, A. E., Koester, S., Goulding, M. and Lassar, A. B. (1997). Ectopic Pax-3 activates MyoD and Myf-5 expression in embryonic mesoderm and neural tissue. Cell 89, 139-148.

Münsterberg, A. E. and Lassar, A. B. (1995). Combinatorial signals from the neural tube, floor plate and notochord induce myogenic bHLH gene expression in the somite. Development 121, 651-660.

Münsterberg, A. E., Kitajewski, J., Bumcrot, D. A., McMahon, A. P. and Lassar, A. B. (1995). Combinatorial signaling by Sonic hedgehog and Wnt family members induces myogenic bHLH gene expression in the somite. Genes Dev. 9, 2911-2922.

Nellen, D., Burke, R., Struhl, G. and Basler, K. (1996). Direct and long-range action of a DPP morphogen gradient. Cell $\mathbf{8 5}, 357-368$.

Ordahl, C. P. and Le Douarin, N. M. (1992). Two myogenic lineages within the developing somite. Development 114, 339-353.

Piccolo, S., Sasai, Y., Lu, B. and De Robertis, E. M. (1996). Dorsoventral patterning in Xenopus: inhibition of ventral signals by direct binding of chordin to BMP-4. Cell 86, 589-598.

Pourquie, O., Coltey, M., Teillet, M. A., Ordahl, C. and Le Douarin, N. M. (1993). Control of dorsoventral patterning of somitic derivatives by notochord and floor plate. Proc. Natl. Acad. Sci. USA 90, 5242-5246.

Pourquie, O., Coltey, M., Breant, C. and Le Douarin, N. M. (1995). Control of somite patterning by signals from the lateral plate. Proc. Natl. Acad. Sci. USA 92, 3219-3223.

Pourquie, O., Fan, C. M., Coltey, M., Hirsinger, E., Watanabe, Y., Breant, C., Francis-West, P., Brickell, P., Tessier-Lavigne, M. and Le Douarin, N. M. (1996). Lateral and axial signals involved in avian somite patterning: a role for BMP4. Cell 84, 461-471.

Pownall, M. E., Strunk, K. E. and Emerson, C. P. J. (1996). Notochord signals control the transcriptional cascade of myogenic bHLH genes in somites of quail embryos. Development 122, 1475-1488.

Riddle, R. D., Johnson, R. L., Laufer, E. and Tabin, C. (1993). Sonic hedgehog mediates the polarizing activity of the ZPA. Cell 75, 1401-1416.

Rong, P. M., Teillet, M. A., Ziller, C. and Le Douarin, N. M. (1992). The neural tube/notochord complex is necessary for vertebral but not limb and body wall striated muscle differentiation. Development 115, 657-672.

Sasai, Y., Lu, B., Steinbeisser, H., Geissert, D., Gont, L. K. and De Robertis, E. M. (1994). Xenopus chordin: a novel dorsalizing factor activated by organizer- specific homeobox genes. Cell 79, 779-790.

Selleck, M. A. and Stern, C. D. (1991). Fate mapping and cell lineage analysis of Hensen's node in the chick embryo. Development 112, 615-626.

Smith, W. C. and Harland, R. M. (1992). Expression cloning of noggin, a new dorsalizing factor localized to the Spemann organizer in Xenopus embryos. Cell 70, 829-840.

Smith, W. C., Knecht, A. K., Wu, M. and Harland, R. M. (1993). Secreted noggin protein mimics the Spemann organizer in dorsalizing Xenopus mesoderm. Nature 361, 547-549.

Stern, H. M., Brown, A. M. and Hauschka, S. D. (1995). Myogenesis in paraxial mesoderm: preferential induction by dorsal neural tube and by cells expressing Wnt-1. Development 121, 3675-3686.

Tonegawa, A., Funayama, N., Ueno, N. and Takahashi, Y. (1997). Mesodermal subdivision along the mediolateral axis in chicken controlled by different concentrations of BMP-4. Development 124, 1975-1984.

Zimmerman, L. B., De Jesus-Escobar, J. M. and Harland, R. M. (1996). The Spemann organizer signal noggin binds and inactivates bone morphogenetic protein 4. Cell 86, 599-606.

(Accepted 12 September 1997) 